\title{
Towards multi-purpose IS radar experiments
}

\author{
I. I. Virtanen ${ }^{1}$, M. S. Lehtinen ${ }^{2}$, and J. Vierinen ${ }^{2}$ \\ ${ }^{1}$ Department of Physical Sciences, University of Oulu, P.O. Box 3000, 90014, Finland \\ ${ }^{2}$ Sodankylä Geophysical Observatory, 99600, Sodankylä, Finland
}

Received: 20 December 2007 - Revised: 17 March 2008 - Accepted: 4 June 2008 - Published: 5 August 2008

\begin{abstract}
The EISCAT incoherent scatter radars routinely perform simultaneous measurements of E- and F-regions of the ionosphere. In addition several experiments exist for measuring pulse-to-pulse correlations from the D-region. However, the D-region experiments have quite limited range extents and the short lags suffer from F-region echoes, which are difficult to properly handle with standard decoding methods.

In this paper it is demonstrated with real data how Dregion experiments can be designed to produce continuous lag profiles extending above the F-region maximum. The large range coverage is attained for all lags shorter than the longest transmission pulse and it allows one to properly include the F-region echoes in the analysis. The large coverage is not needed for pulse-to-pulse lags because E- and F-regions do not have this long correlation times. The lag profiles with large range extent also provide a useful measurement of the upper parts of the ionosphere.

The experiments utilise new kind of phase coding technique, which has estimation accuracy comparable to that of alternating codes though the code sequence is very short. No special decoding method is applied to the codes, because the lag profile inversion method automatically adapts to any kind of transmission codes provided transmission samples are available.

The computing resources needed for real-time lag profile inversion with two different kinds of goals are also discussed here: 1) real-time monitoring of the results and 2) use of inverted lag profiles as a way to permanently store the data. While it was possible to accomplish real-time monitoring with a standard high-end desktop workstation, the higher resolution requirement for permanent data storage purposes is a much more critical task, requiring the use of larger-scale parallel processing.
\end{abstract}

Keywords. Radio science (Ionospheric physics; Signal processing; Instruments and techniques)

Correspondence to: I. I. Virtanen

(ilkka.i.virtanen@oulu.fi)

\section{Introduction}

A common way to arrange transmission pulses in incoherent scatter radar (ISR) experiments is to transmit pulses of equal length with equal inter pulse periods (IPP). Different decoding methods are easy to apply with these arrangements, if the received echoes are assumed to originate from a single scattering pulse at a time. This assumption limits the range coverage of experiments to one IPP. Even if the assumption of a single scattering pulse at a time is relaxed, a "dead" range interval from which no echoes are received is caused by the equal IPPs. In case of D-region pulse-to-pulse measurements the IPPs need to be short to attain a sufficient lag resolution. Thus the range extent of these experiments has been quite limited and F-region echoes from earlier transmitted pulses are difficult to properly take into account (e.g. Turunen et al., 2002). If different code sequences are included in same experiment, they are usually transmitted on their own frequency channels and the decoding of each channel is performed as a separate process. Because the echoes from pulses centered at different frequencies do not correlate, pulse-to-pulse lags can not be measured between the different code sequences.

In this paper it is demonstrated with real data how several different code sequences can be transmitted in the same frequency channel with short unequal IPPs. By using only a single frequency channel a possibility to calculate pulse-topulse correlations between all transmitted pulses is attained. Because the IPPs are of unequal length, the first "dead" range can be easily pushed to more than thousand kilometers away from the radar. With these arrangements continuous lag profiles from D-region to above F-region maximum can be measured at lag values shorter than the longest pulse. This naturally means that the received signal is a sum of several echoes from pulses at different ranges.

Whereas this kind of situation is rather difficult to handle with traditional decoding methods, for statistical inversion based lag profile inversion (Virtanen et al., 2008) it does not differ from analysis of standard experiments. 


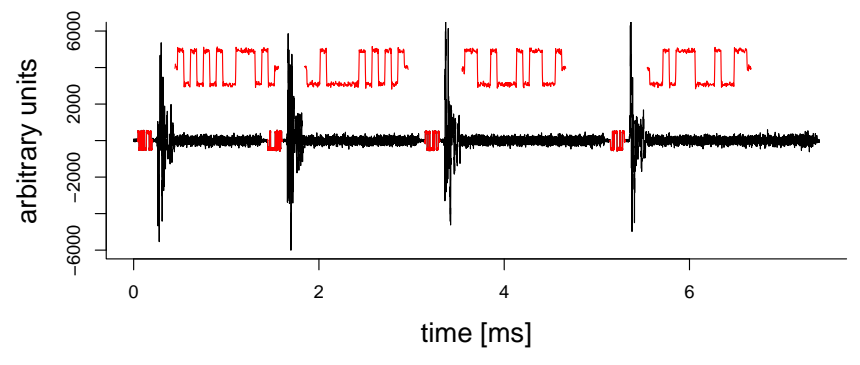

Fig. 1. Raw data of a full transmission cycle from experiment 1 . Transmission signals are marked with red color. After each pulse a larger image of the transmission envelope is plotted above the received signal.

To allow data analysis with range resolution better than the transmission pulse length, radar codes (transmission modulations) of incoherent scatter radar experiments are usually selected to fulfill certain decoding properties. However, the need of a special decoding method can be removed with lag profile inversion, which allows any phase code to be analysed with desired range-, lag- and time-resolutions. Though the inversion is possible with any phase code or a sequence of codes, the estimation accuracy of an experiment is much dependent on the codes used. When introducing the present lag profile inversion software (Virtanen et al., 2008), the possibility to search for efficient radar codes without taking care of the decoding properties (Vierinen et al., 2008) was mentioned, but only alternating code (Lehtinen and Häggström, 1987) experiments were used to allow comparison between the two analysis methods. The lag profile inversion analysis of short code sequences has been earlier used at the EISCAT Svalbard radar by Lehtinen et al. (2002). The experiments used in this paper are the first ever utilising a new phase coding techique, which has estimation accuracy comparable to that of alternating codes, though the code cycle is very short. In this paper code sequences consisting of four different phase coded pulses are used, which is the reason to use the term "code quad" when referring to these codes. The method for evaluating different code sequences is introduced in another paper in this issue (Lehtinen et al., 2008), where the code quads are also compared to other kinds of radar coding techniques.

\section{New experiments}

In this paper two experiments selected from several test runs performed during the finnish EISCAT campaign on October 2007 are introduced. The first of the experiments, hereafter referred as "experiment 1", was run with the UHF radar. Its D-region capabilities are mainly limited to the short lags, none of which significantly differs from power profile at D-
Table 1. Phase codes used in the experiments.

\begin{tabular}{|c|c|c|c|c|c|c|c|c|c|c|c|c|c|c|}
\hline \multicolumn{15}{|c|}{ 15-bit code quad } \\
\hline+ & - & + & - & + & - & + & - & - & + & + & + & - & + & - \\
\hline- & - & + & - & - & - & - & - & + & - & + & - & + & - & + \\
\hline+ & + & - & - & + & - & - & - & + & - & + & + & - & - & + \\
\hline- & - & + & - & + & + & + & - & - & - & + & - & - & + & + \\
\hline & & & & \multicolumn{7}{|c|}{ 4-bit weak alternating code } & & & & \\
\hline & & & & - & & - & & + & & - & & & & \\
\hline & & & & - & & + & & - & & - & & & & \\
\hline & & & & + & & - & & - & & + & & & & \\
\hline & & & & + & & + & & + & & + & & & & \\
\hline
\end{tabular}

\begin{tabular}{|c|c|c|c|c|c|c|c|c|c|c|c|c|}
\hline \multicolumn{13}{|c|}{ 13-bit Barker code } \\
\hline+ & - & + & - & + & + & - & - & + & + & + & + & + \\
\hline
\end{tabular}

region altitudes. However, the pulses are sufficiently short for measuring the power profile from quite low ranges.

The second experiment, hereafter referred as "experiment 2", was run with the VHF radar. It provides a large number of useful pulse-to-pulse lags in addition to the short ones. The full code cycle of the experiment contains 16 phase coded pulses with varying bit and pulse lengths. More detailed information about the experiments is given in the following sections.

\subsection{Experiment 1}

Experiment 1 utilises one phase code sequence, the 15-bit code quad in Table 1. Bit length of the codes is $10 \mu$ s leading to total pulse length of $150 \mu \mathrm{s}$. The selected bit length makes the experiment optimal for roughly $1 \mathrm{~km}$ target range resolution (Lehtinen, 1989). This does not mean that structures smaller or larger than $1 \mathrm{~km}$ could not be seen, because the estimation accuracy drops relatively slowly as the target resolution changes to either direction. The experiment was recorded as raw data with $2 \mathrm{MHz}$ sampling frequency, which theoretically allows analysis with $75 \mathrm{~m}$ range resolution.

The four pulses in the code quad are transmitted with unequal IPPs, i.e. there is a different receiving time after each pulse in the quad. The IPPs, measured from start of a pulse to start of the next one, are 1400,1700, 2000 and $2300 \mu$ s. Real part of raw data from one full code cycle is plotted in Fig. 1.

The recorded signal is a sum of echoes from several pulses at different ranges. Each signal sample has contribution from all ranges covered by transmitted pulses at the particular instant of time when the sample was recorded. Because echoes can not be received during transmissions, the combination of IPPs and pulse lengths defines the number of recorded 

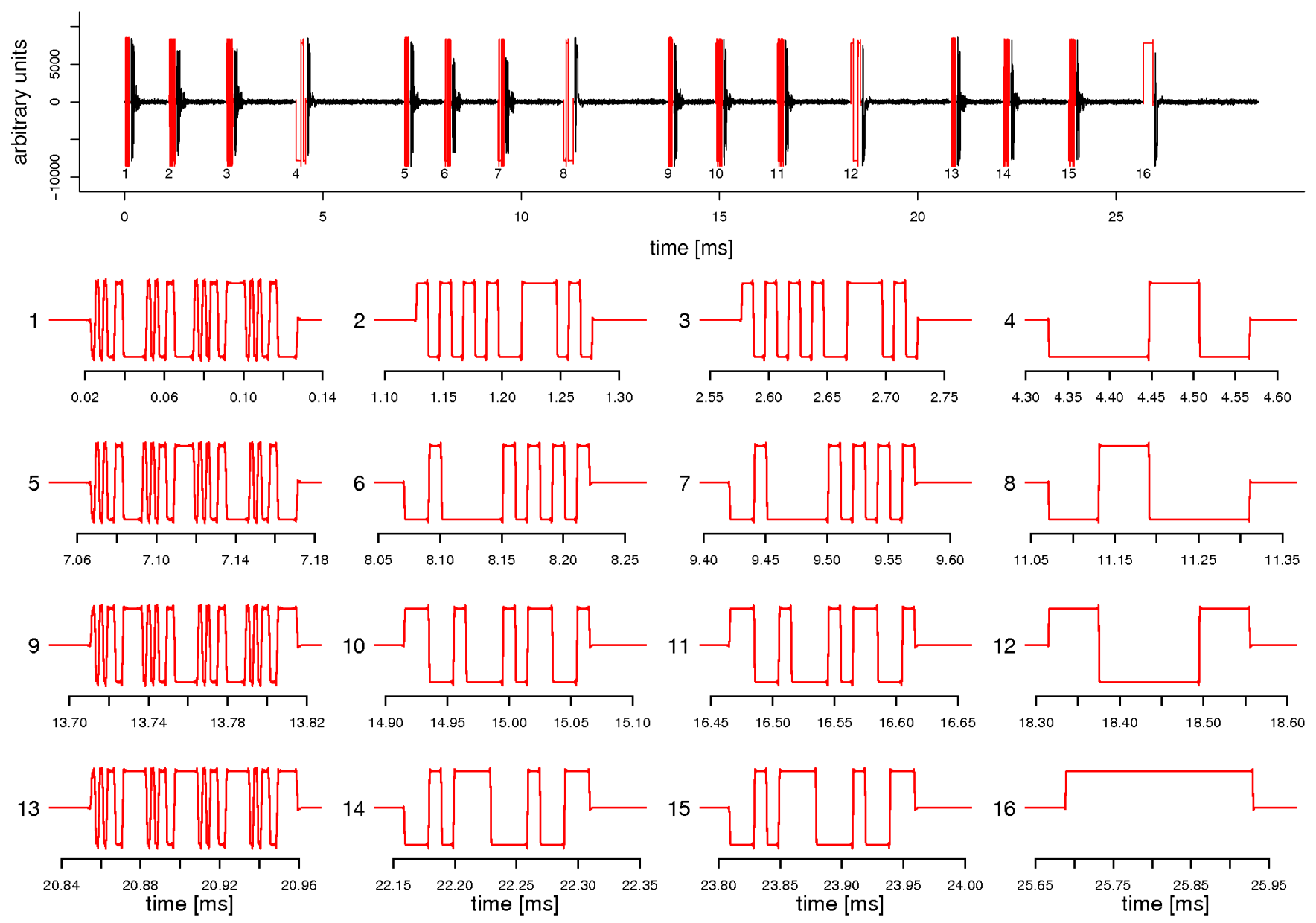

Fig. 2. Top panel: Raw data of a full transmission cycle from experiment 2. Transmission signals are marked with red color. Lower panels: Larger images of the numbered transmission envelopes, notice the differing time scales.

samples with contribution from each range. To make comparison of different experiment easier, we use the concept of radar efficiency: the number of echo samples from each range is normalized using the total duration of the experiment and the maximum duty cycle of the radar so that the largest possible efficiency is 1.0 , meaning that the radar duty cycle is fully occupied and all echoes are received. Values smaller than one mean either that the duty cycle is not fully occupied or echoes are lost during subsequent transmissions. The radar efficiency for experiment 1 as a function of range is plotted in Fig. 4.

There are certain technical limitations causing the efficiency values clearly smaller than one: The clystron beam must be turned on $40 \mu$ s before the start of an actual pulse and there is an upper limit of $12.6 \%$ for the beam duty cycle. From these numbers we can calculate that the true maximum RF duty cycle for $150 \mu$ s pulses is $10 \%$ corresponding the value 0.8 in radar efficiency. In fact the maximum RF duty cycle of the EISCAT UHF radar (12.4\%) can only be achieved with the maximum allowed pulse length (2000 $\mu$ s). The efficiency value is not constant because some echoes are always lost during the transmissions of the subsequent pulses. This behavior is not a specific weakness of the new experiments, similar deviations from the maximum value would result also if several frequency channels would be used to fill the radar duty cycle.

\subsection{Experiment 2}

Experiment 2 can be seen as an extended version of experiment 1 . Its code cycle consists of 16 different phase coded pulses, among which three different bit lengths are used. The code cycle is constructed by combining four sets of four phase coded pulses. The properties of the code sets are listed in Table 2.

In the experiment the first codes of each set are first transmitted one after the other, then the second ones etc. All the IPPs between adjacent pulses are of different lengths, starting from the IPP between the first codes of set 1 and set 2 the 


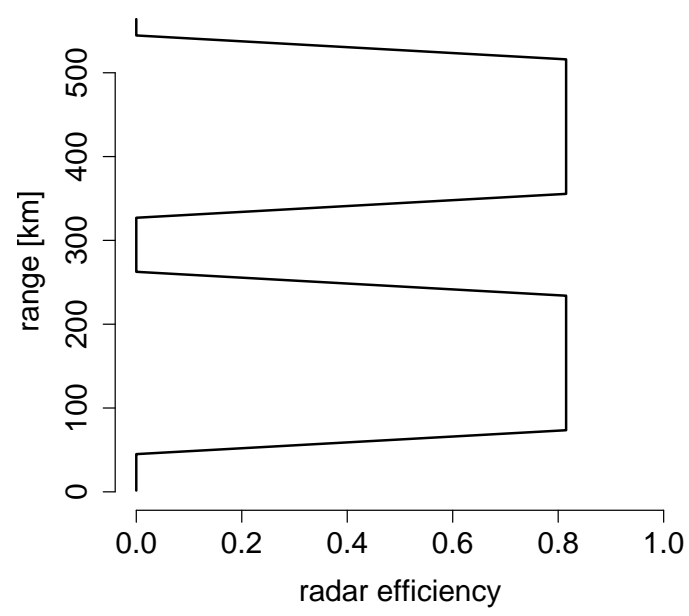

Fig. 3. Radar efficiency for standard EISCAT UHF experiment "manda". Here efficiency value 1.0 would mean that the maximum duty cycle of $12.4 \%$ is fully used. Though the experiment is analysed up to larger ranges than the length of one IPP, a "dead" range is produced around $300 \mathrm{~km}$ from which no echoes are received.

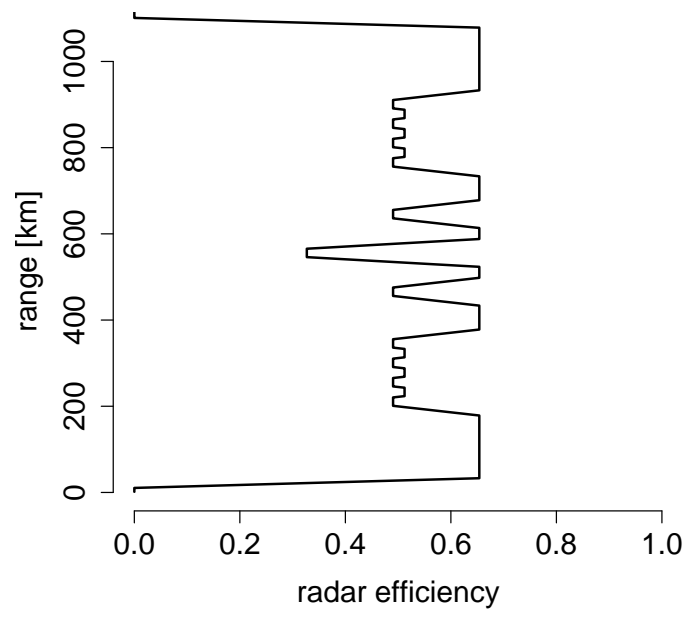

Fig. 4. Radar efficiency for experiment 1 introduced in this paper.

IPPs are 1104, 1450, 1750, 2740, 1004, 1350, 1650, 2640, $1204,1550,1850,2540,1304,1650,1880$ and $2934 \mu$ s. Real part of raw data from a full code cycle is plotted in Fig. 2.

According to Lehtinen (1989), the bit lengths of the code sets 1, 2 and 4 are optimal for roughly $200 \mathrm{~m}, 1 \mathrm{~km}$ and $6 \mathrm{~km}$ target resolutions, respectively (set 3 is exactly the same as set 2). Moreover, the pulse-to-pulse and fractional lags are supposed to be optimal for target resolution corresponding the shorter bit lengths, as the optimal target resolution is determined by the Fourier components of the range ambiguity function (Lehtinen, 1989). The radar efficiencies for each different code and the total efficiency are plotted in Fig. 5. Despite the short IPPs the theoretical range extent of the experiment is very large.

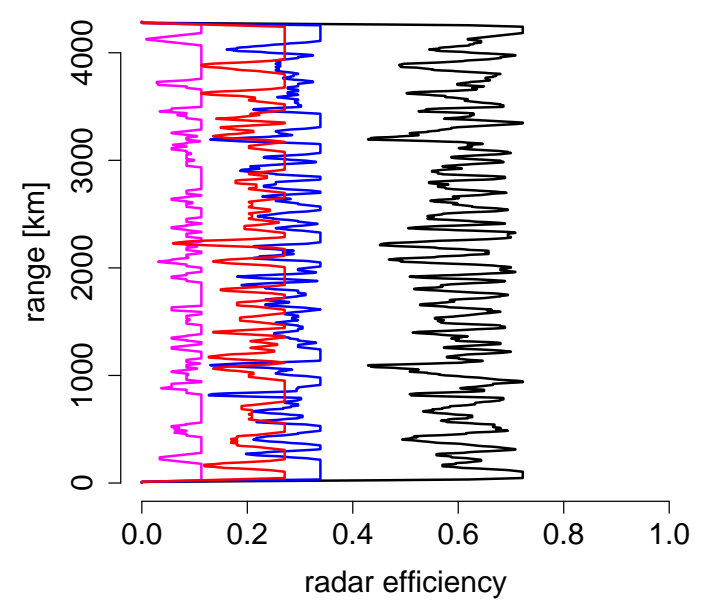

Fig. 5. Radar efficiency for experiment 2 introduced in this paper. Efficiency of the code with $2 \mu$ s (magenta), $10 \mu$ s (blue) and $60 \mu \mathrm{s}$ (red) bits are drawn as separete curves. The black curve is the total efficiency.

Table 2. Code sets used in experiment 2. If Barker codes are used the bit length in fourth column is that of the Barker code.

\begin{tabular}{cccc}
\hline set number & code set & $\begin{array}{c}\text { sub-bit } \\
\text { Barker code }\end{array}$ & bit length $[\mu \mathrm{s}]$ \\
\hline set 1 & 4-bit weak alternating code & 13-bit & 2 \\
set 2 & 15-bit code quad & - & 10 \\
set 3 & Same as set 2 & - & 10 \\
set 4 & 4-bit weak alternating code & - & 60 \\
\hline
\end{tabular}

\section{Analysis results}

Both short lags (shorter than longest pulse length) and Dregion pulse-to-pulse lags were inverted from recorded raw data using lag profile inversion software described in (Virtanen et al., 2008). The resolutions used in the following examples are just one possible choice, the combination of raw data and lag profile inversion gives the user a freedom to select the resolutions after running the experiment and also to analyse the same data with different resolutions.

For experiment 1 the lag profile inversion was performed with $5 \mathrm{~s}$ time resolution, $5 \mu \mathrm{s}$ lag resolution and range resolution varies from $1.5 \mathrm{~km}$ to $50 \mathrm{~km}$. The range-gates were chosen to cover also the strong ionization of the F-region. An example plasma scattering autocorrelation function (ACF) resulting from the lag profile inversion is plotted in the left panel of Fig. 6, where all the main regions of ionosphere are clearly visible. In addition a sporadic-E layer occurred at about $107 \mathrm{~km}$ range. After the lag profile inversion, plasma parameters can be fitted to the ACF estimates. An example 

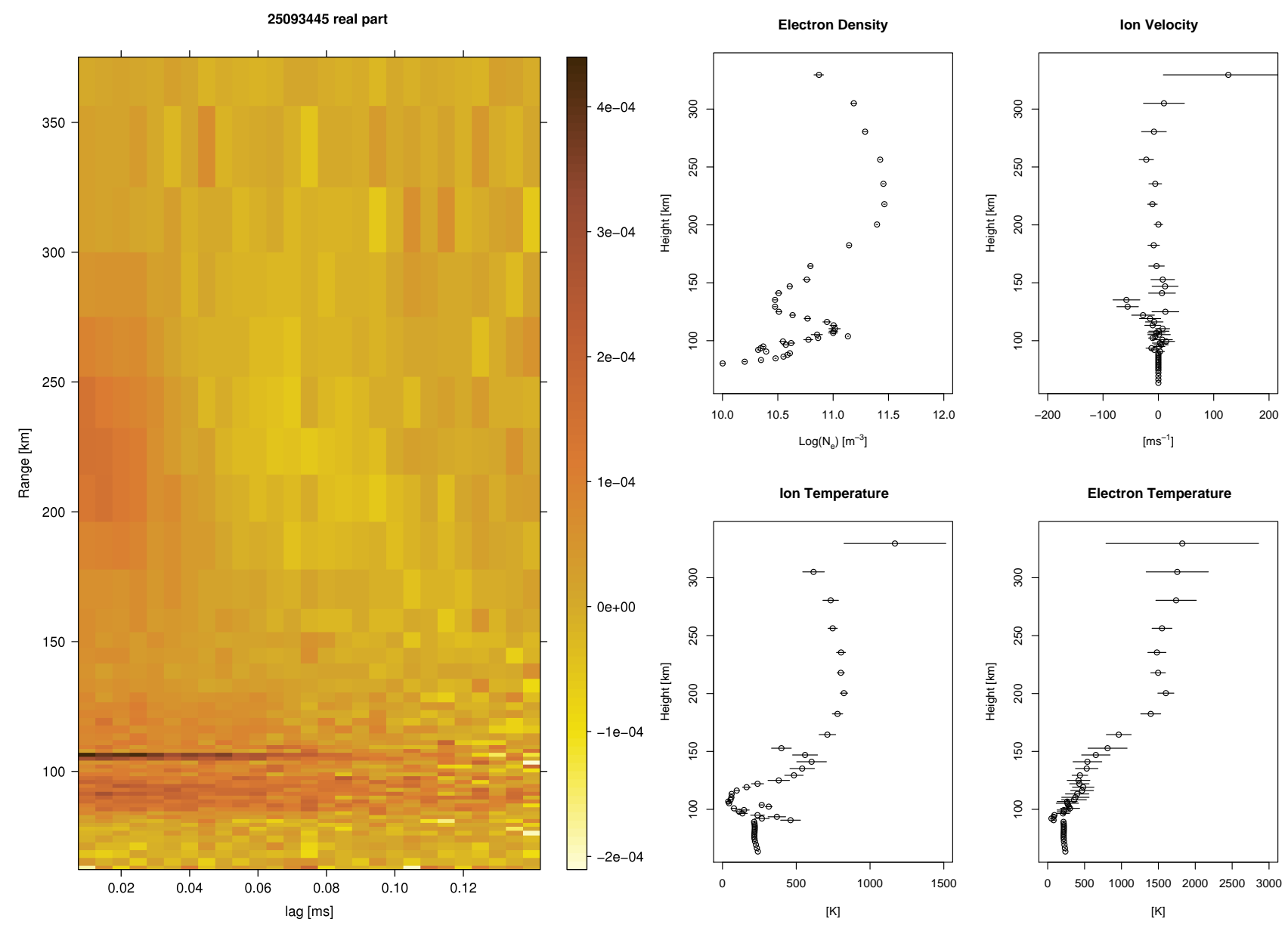

Fig. 6. Analysis results of experiment 1 from 18 October 2007 10:24 UT. Left panel: Real part of ACF in arbitrary units. Time resolution is $5 \mathrm{~s}$ and range resolution varies from $1.5 \mathrm{~km}$ in D- and lower E-regions to $50 \mathrm{~km}$ above F-region peak. Right panel: Plasma parameters with $1 \mathrm{~min}$ integration time. The horisontal lines are $68 \%$ confidence intervals. Model values are used for ion mass and collision frequency at all altitudes, for ion temperature and electron to ion temperature ratio below $90 \mathrm{~km}$ and ion velocity is set to zero below $90 \mathrm{~km}$. Electron density is not calibrated.

of a four-parameter fit with 1 min time resolution is plotted in right panel of Fig. 6.

An attempt was also made to invert D-region pulse-topulse lags from the data, but as assumed the echoes do not correlate long enough for reasonable pulse-to-pulse measurements. (This is usually the case with UHF experiments. A VHF version of this experiment is expected to produce useful pulse-to-pulse data, but the VHF version was not run this time.) Anyhow, the D-region power profile was successfully measured.

Experiment 2 was analysed with the same range- and time-resolutions as experiment one. Because the shortest bit length in the experiment was only $2 \mu$ s, the data can not be decimated to longer sampling interval than this. On the other hand, because the ACFs of the VHF radar are about four times as long as those of the UHF radar, even lower lag resolution than that used for experiment 1 could be used. For these reasons several fractional lags were included in each main lag to produce the $25 \mu$ s lag resolution seen in the left panel of Fig. 7. A similar four-parameter fit as performed for experiment 1 was also made for the experiment 2, the result of the parameter fit is seen in the right panel of Fig. 7. Though the lag profiles were successfully inverted, it is evident that the pulses in experiment 2 were too short for measuring ion and electron temperatures at $\mathrm{E}$ and lower $\mathrm{F}$ regions. From the ACF plot it is seen that below $200 \mathrm{~km}$ even the first zero of the ACF is not meat, which leads to doubtful fit results for the temperatures.

An example of the pulse-to-pulse lags is plotted in Fig. 8. The experiment timing scheme allows very many pulse-to-pulse lags to be inverted. Many of them are so near to each other that they can be safely included in same lag profile at D-region altitudes. The data was analysed by dividing all lag values from $1 \mathrm{~ms}$ to $20 \mathrm{~ms}$ into $1 \mathrm{~ms}$ slides. All 

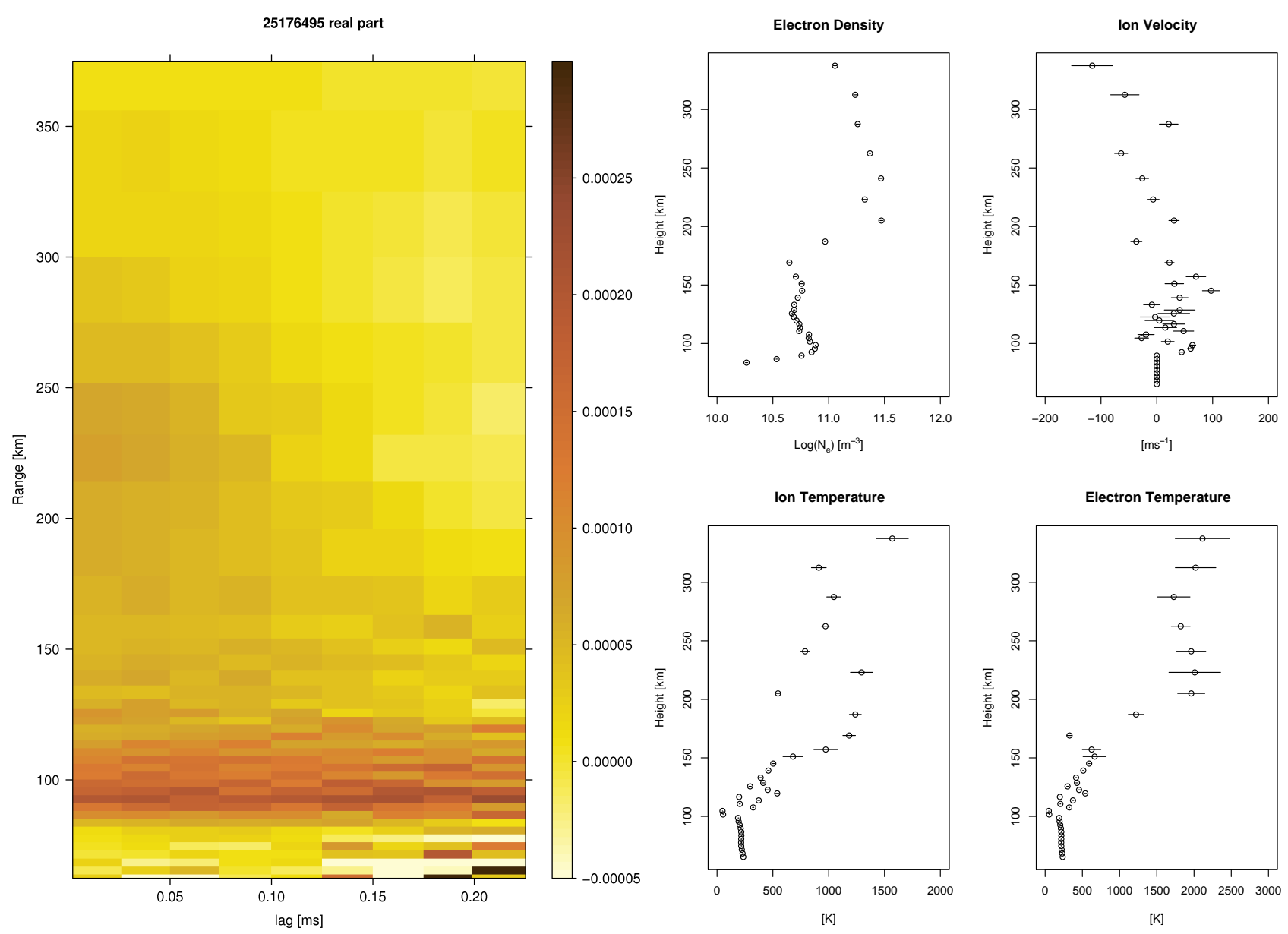

Fig. 7. Analysis results of experiment 2 from 19 October 2007 09:28 UT. Left panel: Real part of ACF in arbitrary units. Time resolution is $5 \mathrm{~s}$ and range resolution varies from $3 \mathrm{~km}$ in D- and lower E-regions to $50 \mathrm{~km}$ above F-region peak. Right panel: Plasma parameters with 1 min time resolution. The horisontal lines are $68 \%$ confidence intervals. Model values are used for ion mass and collision frequency at all altitudes, for ion temperature below $100 \mathrm{~km}$, for electron to ion temperature ratio below $150 \mathrm{~km}$ and ion velocity is set to zero below $90 \mathrm{~km}$. Due to insufficiently short pulses of the experiment the fit results for temperatures may not be reliable. Electron density is not calibrated.

lags inside each slide were combined in one lag profile, i.e. the final lag resolution of the analysis is $1 \mathrm{~ms}$. The analysis program tests all lag values and automatically drops off those ones which do not have any non-zero parts in range ambiguity function. In this way different lag values may get different estimation accuracies as the lag profiles are combinations of varying number of fractional lags, which is readily seen in the variances of the inversion results. For the user this kind of analysis is particularly easy, as no information of the actual IPPs is needed.

We want to specially emphasize the fact that all kinds of lagged products are included in the inversion, especially also those resulting from correlation between the Barker coded alternating codes and the plain alternating codes, as well as the products resulting from correlation between the Barker coded alternating codes and the 15-bit code quads. Also the fractional lags between the 15 -bit code quads contribute significantly to the high-resolution estimates. Moreover, of course even the not-so-fractional (close to full) lags contribute, even if their ambiguity functions consist of bits wider than the desired resolution. Everything is automatically included, correctly weighted, by the inversion process. All this is omitted in a standard type of experiment, where different code sequences are on different frequencies and they cannot be correlated with each other.

As the criteria used for selecting the code sequences assumes only one scattering pulse at a time, the quality of the new experiments was not exactly known beforehand. On the basis of Lehtinen et al. (2008) we do know that the code sequences should perform well in a standard type of experiment with equal IPPs. In data analysis the lag profile variances offered by lag profile inversion are used in the parameter fit. This way the estimation accuracy of the experiments is taken into account, but the analysis does not offer 
anything that could be directly used for comparisons with other experiments. Numerical studies of the variance behavior much similar to those presented in Lehtinen et al. (2008) could be performed using lag profile inversion. This kind of analysis will be important when designing future experiments, but in this paper we settle on demonstrating that we are able to analyse the new experiments. If the phase codes used would perform perfectly with the unequal IPPs and if the assumption of small signal to noise ratio is justified, the ratio of best attainable accuracy and that attained from the new experiments would follow the radar efficiency curves in Figs. 4 and 5. Here one should notice that the efficiency 1.0 is very difficult to achieve in practical experiments as mentioned in Sect. 2.1.

\section{Resources needed for real-time lag profile inversion}

The experiments used in this paper were both analysed in real-time using lag profile inversion, but with too low resolutions to be used for storing the data. The analysis task was shared with eight processors without any special parallel processing - the parallelization was accomplished at the UNIX command line level by generating independent command-line analysis processes accessing different pieces of data. These commands were generated automatically by R scripts (R Development Core Team, 2007), which was also used for visualization and user interface. The analysis speed was also increased by calculating averages of raw lag profiles before the inversion, which is not the most accurate way of performing the analysis. The results in this paper were later calculated without the averaging. Here some numbers are shown of the computing resources needed for high-resolution lag profile inversion in real-time.

The resolutions needed for the stored lag profiles are very much dependent on the experiment and its target. In the following calculations it is assumed that an integration time of $2 \mathrm{~s}$ is always used. Then range extent, number of range gates and data decimation are varied. The number of floating point operations needed for the analysis (FLOP count) and the CPU time used by the current analysis computer $(8 \times 3 \mathrm{GHz}$ Eight-Core Intel Xeon, 16 GB 667 MHz DDR2 FB-DIMM, Apple Mac Pro) are measured. An auto-vectorized 64-bit executable using 32-bit floating point precision was built with Intel Fortran version 10.0.

The factors that can affect the FLOP count and CPU time are assumed to be the amount of decimation of the amplitudedomain data, the number of range gates and the range extent of the analysis. Also the experiment timing has effect to the analysis time, because the FLIPS package (Orispää and Lehtinen, $2008^{1}$ ) can solve the problem faster if there are many zeros in the beginning or end of the theory matrix rows. In this sense having several pulses simultaneously in

\footnotetext{
${ }^{1}$ Orispää, M. and Lehtinen, M. S.: Fortran Linear Inverse Problem Solver (FLIPS), unpublished manuscript, 2008.
}

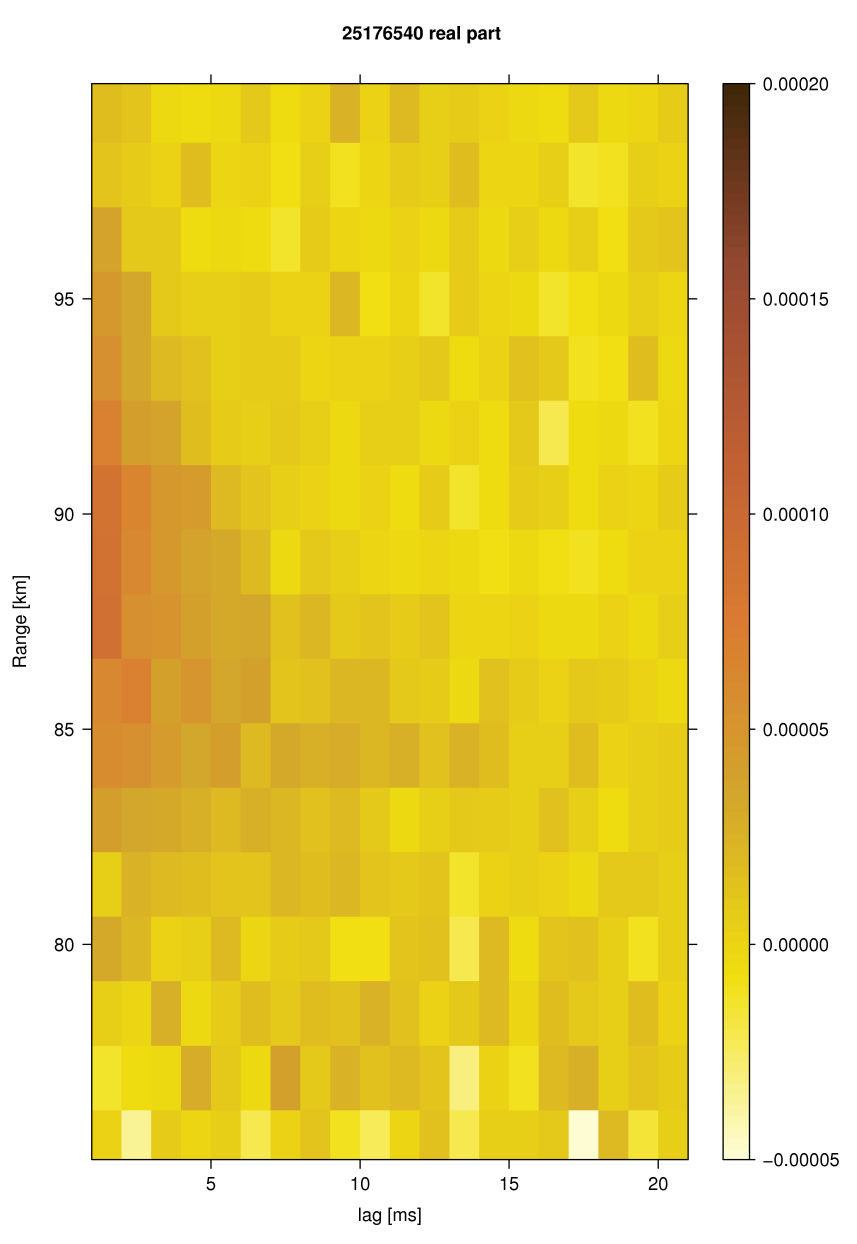

Fig. 8. Real parts of some D-region pulse-to-pulse lag profiles from experiment 219 October 2007 09:28 UT. The time resolution is $1 \mathrm{~min}$, lag resolution $1 \mathrm{~ms}$ and range resolution $1.5 \mathrm{~km}$. The ACF is in arbitrary units, but in same units as the ACF in Fig. 7.

ionosphere is not optimal situation for lag profile inversion, since it leads to theory matrix rows with only few zeros, if any, in both ends.

In Table 3 there are some numbers from test runs with the experiment 2 introduced in this paper. As high range resolutions (of the order of $100 \mathrm{~m}$ ) are sometimes needed in lower ranges, the data can not be decimated much when analysed for storage purposes. On the fifth row is the combination of the nearest values that could be used in routine storage. The other numbers are calculated to see how the software behaves as the parameters are varied.

Using the numbers in Table 3 it can be calculated that inverting 100 lag profiles with $150 \mathrm{~m}$ maximum range resolution and in total 500 range gates requires about $140 \mathrm{TFLOP} / \mathrm{s}$ with the current software. With similar machinery as used in the test this would mean about 40000 cores or 5000 computers working simultaneously. 
Table 3. Computing power needed for selected resolutions. All numbers are for one lag profile starting from $50 \mathrm{~km}$ range. Integration time is $2 \mathrm{~s}$. The CPU times are for one core of an $8 \times 3 \mathrm{GHz}$ Eight-Core Intel Xeon desktop computer, $16 \mathrm{~GB} 667 \mathrm{MHz}$ DDR2 FB-DIMM (Apple Mac Pro). The theoretically maximum possible $\mathrm{FLOP} / \mathrm{s}$ rate of such a core is 4 times the CPU frequency assuming the vector SIMD SSE unit can operate at full speed making four 32-bit vector floating point operations each clock cycle. Thus the theoretical speed being 1.6e10 FLOP/s and the actual speed being about a quarter of that in many cases can be considered very satisfactory.

\begin{tabular}{cccccc}
\hline $\begin{array}{c}\text { Number } \\
\text { of gates }\end{array}$ & $\begin{array}{c}\text { max } \\
\text { range }[\mathrm{km}]\end{array}$ & $\begin{array}{c}\text { sampling } \\
\text { interval }[\mu \mathrm{s}]\end{array}$ & $\begin{array}{c}\text { FLOP } \\
\text { count }\end{array}$ & $\begin{array}{c}\text { CPU } \\
\text { time [s] }\end{array}$ & FLOP/s \\
\hline 500 & 2000 & 10.0 & $2.8 \mathrm{e} 11$ & 76 & $3.6 \mathrm{e} 9$ \\
500 & 2000 & 5.0 & $5.5 \mathrm{e} 11$ & 147 & $3.7 \mathrm{e} 9$ \\
500 & 2000 & 2.5 & $1.1 \mathrm{e} 12$ & 291 & $3.8 \mathrm{e} 9$ \\
500 & 2000 & 1.0 & $2.8 \mathrm{e} 12$ & 717 & $3.9 \mathrm{e} 9$ \\
400 & 2000 & 10.0 & $1.7 \mathrm{e} 11$ & 51 & $3.4 \mathrm{e} 9$ \\
300 & 2000 & 10.0 & $1.0 \mathrm{e} 11$ & 33 & $3.1 \mathrm{e} 9$ \\
200 & 2000 & 10.0 & $4.5 \mathrm{e} 10$ & 18 & $2.5 \mathrm{e} 9$ \\
100 & 2000 & 10.0 & $1.2 \mathrm{e} 10$ & 8.5 & $1.4 \mathrm{e} 9$ \\
50 & 2000 & 10.0 & $3.2 \mathrm{e} 9$ & 5.1 & $6.3 \mathrm{e} 8$ \\
400 & 1601 & 10.0 & $1.7 \mathrm{e} 11$ & 51 & $3.4 \mathrm{e} 9$ \\
300 & 1220 & 10.0 & $9.3 \mathrm{e} 10$ & 31 & $3.0 \mathrm{e} 9$ \\
200 & 830 & 10.0 & $3.8 \mathrm{e} 10$ & 16 & $2.3 \mathrm{e} 9$ \\
100 & 441 & 10.0 & $7.6 \mathrm{e} 9$ & 6.8 & $1.1 \mathrm{e} 9$ \\
50 & 244 & 10.0 & $1.4 \mathrm{e} 9$ & 4.0 & $3.4 \mathrm{e} 8$ \\
500 & 1500 & 1.0 & $2.7 \mathrm{e} 12$ & 690 & $3.9 \mathrm{e} 9$ \\
500 & 1000 & 1.0 & $2.5 \mathrm{e} 12$ & 633 & $3.9 \mathrm{e} 9$ \\
500 & 500 & 1.0 & $1.9 \mathrm{e} 12$ & 490 & $3.9 \mathrm{e} 9$ \\
\hline
\end{tabular}

If raw data is stored and real-time analysis is used only for monitoring the ionosphere, much larger range gates and a smaller number of lags can be used. Using the table we can calculate that 20 lag profiles with 100 range gates, $1.5 \mathrm{~km}$ maximum resolution and $2 \mathrm{~s}$ integration time requires about 240 GFLOPs. This is accomplished in $170 \mathrm{~s}$ with one processor. In other words, this kind of real-time monitoring requires $120 \mathrm{GFLOP} / \mathrm{s}$ which can be achieved using about 85 cores or eleven computers. In many cases even much smaller number of range gates is enough for monitoring purposes.

It may seem that analysing the recorded raw data at higher resolutions would be a huge effort. Here one needs to notice that if lag profiles are stored they need to have high resolution, but this is only to be prepared for unusual ionospheric conditions. Raw data contains the necessary information for high-resolution analysis, but for most parts of the data much lower resolutions are sufficient. The small fraction of the data that needs high-resolution analysis can usually be selected from the lower resolution results thus avoiding unnecessary calculations.

\section{Storage resources}

In the previous section we estimated the computational resources necessary for real-time inversion of lag profile data and found that while it is perfectly possible to do real-time inversion for monitoring purposes, inversion for resolutions good enough to facilitate use of inverted lag profile data for further analysis requires very much in terms of computational power. While this kind of computational power might still be a reasonable requirement for a future incoherent scatter facility, we feel it is useful to consider storage of raw (uncorrelated) amplitude-domain data instead of the storage of lag profiles.

The analysis in this paper is based on baseband detected complex data. The sampling frequency was $2 \mathrm{MHz}$ facilitating $75 \mathrm{~m}$ range resolution throughout the ionosphere and arbitrarily high time resolution. The possible lag values are limited by the selected pulse lengths and IPPs, but within these limits a lag resolution of $0.5 \mu$ s can be achieved. On the other hand, by including several lag profiles in same inversion problem arbitrarily low lag resolutions are also possible without losing the high range resolution. In addition there is no limitations for the longest lag. We have routinely used this kind of data storage for finnish special experiments and can presently store 4 Terabytes of data on a cheap commercial RAID disk system, enough for $138 \mathrm{~h}$ of raw amplitudedomain data. The $2 \mathrm{MHz}$ sampling frequency is not enough for recording the plasma lines, but the plasma line information is lost in standard lag profile storage also. To maintain the plasma line information in the data one can either increase the sampling frequency or analyse the plasma lines as separate process in real time using other analysis methods.

\section{Conclusions}

In this paper we have demonstrated how the transmission pulses of incoherent scatter radar experiments can be arranged in a way that allows lag profile analysis with a large range coverage though the pulses are transmitted with short inter pulse periods. The data analysis of this kind of an experiment is also demonstrated with real experimental data. Though the lag profile inversion part of the analysis was successful, the fit of ion and electron temperatures was not very successful with experiment 2 . The problem is easy to solve by including longer pulses in the experiment to allow long enough lags to be inverted. The experiment optimisation in the form of carefully evaluating and minimising the variances of the inverted lag profiles is the natural next step in this work.

We have also considered lag profile inversion results as an alternative way for storing IS radar data and found that rather challenging, while not perhaps impossible, in terms of required computing power. Motivated by this, we suggest the storage of baseband-detected amplitudes of echoes as a fully 
flexible storage format for future IS radars. Also, in terms of actual hardware, saving baseband data is trivial with offthe-shelf equipment. The full (ion line) bandwidth of a radar signal generates about the same data bandwidth as a standard $\mathrm{HD}$ video stream at the time of writing this article.

Acknowledgements. The EISCAT measurements were made with special programme time granted for Finland. EISCAT is an international assosiation supported by China (CRIRP), Finland (SA), Germany (DFG), Japan (STEL and NIPR), Norway (NFR), Sweden (VR) and United Kingdom (PPARC). This work was supported by the Academy of Finland (application number 213476, Finnish Programme for Centres of Excellence in Research 2006-2011; application number 43988, EISCAT Data Analysis and Research) and by the Finnish Graduate School in Astronomy and Space Physics.

Topical Editor K. Kauristie thanks I. Häggström and another anonymous referee

\section{References}

Lehtinen, M., Markkanen, J., Väänänen, A., Huuskonen, A., Damtie, B., Nygrén, T., and Rahkola, J.: A new incoherent scatter technique in the EISCAT Svalbard Radar, Radio Sci., 37, 3-1, doi:10.1029/2001RS002518, 2002.
Lehtinen, M. S.: On optimization of incoherent scatter measurements, Adv. Space Res., 9, 133-141, doi:10.1016/0273-1177(89) 90351-7, 1989.

Lehtinen, M. S. and Häggström, I.: A new modulation principle for incoherent scatter measurements, Radio Sci., 22, 625-634, 1987.

Lehtinen, M. S., Virtanen, I. I., and Vierinen, J.: Fast comparison of IS radar code sequences for lag profile inversion, Ann. Geophys., 26, 2291-2301, 2008, http://www.ann-geophys.net/26/2291/2008/.

R Development Core Team: R: A Language and Environment for Statistical Computing, R Foundation for Statistical Computing, Vienna, Austria, http://www.R-project.org, ISBN 3-900051-070, 2007.

Turunen, T., Westman, A., Häggström, I., and Wannberg, G.: High resolution general purpose D-layer experiment for EISCAT incoherent scatter radars using selected set of random codes, Ann. Geophys., 20, 1469-1477, 2002, http://www.ann-geophys.net/20/1469/2002/.

Vierinen, J., Lehtinen, M. S., Orispää, M., and Virtanen, I. I.: Transmission code optimization method for incoherent scatter radar, Ann. Geophys., accepted, 2008.

Virtanen, I. I., Lehtinen, M. S., Nygrén, T., Orispää, M., and Vierinen, J.: Lag profile inversion method for EISCAT data analysis, Ann. Geophys., 26, 571-581, 2008, http://www.ann-geophys.net/26/571/2008/. 